\title{
Multidimensional trade-offs in species responses to disturbance: implications for diversity in a subtropical forest
}

\author{
María Uriarte,,${ }^{1,5}$ James S. Clark, ${ }^{2}$ Jess K. Zimmerman, ${ }^{3}$ Liza S. Comita, ${ }^{1}$ Jimena Forero-Montaña, ${ }^{3}$ \\ AND JILL THOMPSON ${ }^{3,4}$ \\ ${ }^{1}$ Department of Ecology, Evolution and Environmental Biology, Columbia University, New York, New York 10027 USA \\ ${ }^{2}$ Nicholas School of the Environment and Department of Biology, Duke University, Durham, North Carolina 27708 USA \\ ${ }^{3}$ Institute for Tropical Ecosystem Studies, University of Puerto Rico, Rio Piedras, P.O. Box 23341, \\ San Juan, Puerto Rico 00931-3341 USA \\ ${ }^{4}$ Centre for Ecology and Hydrology-Edinburgh, Bush Estate, Penicuik, Midlothian EH26 OQB United Kingdom
}

\begin{abstract}
Species employ diverse strategies to cope with natural disturbance, but the importance of these strategies for maintaining tree species diversity in forests has been debated. Mechanisms that have the potential to promote tree species coexistence in the context of repeated disturbance include life history trade-offs in colonization and competitive ability or in species' ability to survive at low resource conditions and exploit the temporary resourcerich conditions often generated in the wake of disturbance (successional niche). Quantifying these trade-offs requires long-term forest monitoring and modeling.

We developed a hierarchical Bayes model to investigate the strategies tree species employ to withstand and recover from hurricane disturbance and the life history trade-offs that may facilitate species coexistence in forests subject to repeated hurricane disturbance. Unlike previous approaches, our model accommodates temporal variation in process error and observations from multiple sources. We parameterized the model using growth and mortality data from four censuses of a 16-ha plot taken every five years (1990-2005), together with damage data collected after two hurricanes and annual seed production data (1992-2005).

Species' susceptibilities to hurricane damage as reflected by changes in diameter growth and fecundity immediately following a storm were weak, highly variable, and unpredictable using traditional life history groupings. The lower crowding conditions (e.g., high light) generated in the wake of storms, however, led to greater gains in growth and fecundity for pioneer and secondary-forest species than for shade-tolerant species, in accordance with expectation of life history. We found moderate trade-offs between survival in high crowding conditions, a metric of competitive ability, and long-distance colonization. We also uncovered a strong trade-off between mean species fecundity in low crowding conditions, a metric of recovery potential, and competitive ability. Trade-offs in competitive and colonization ability, in addition to successional niche processes, are likely to contribute to species persistence in these hurricaneimpacted forests. The strategies species employ to cope with hurricane damage depend on the degree to which species rely on sprouting, repair of adult damage, changes in demographic rates in response to enhanced resource availability after storms, or long-distance dispersal as recovery mechanisms.
\end{abstract}

Key words: coexistence; colonization-competition; hierarchical Bayes; hurricanes; life history; Puerto Rico; successional niche; tropical forest diversity.

\section{INTRODUCTION}

A large number of hypotheses to explain tree species coexistence in tropical forests have been put forth and tested (reviewed in Wright 2002). Evidence supports the notion that these communities are open, dynamic systems where natural and human disturbance acting at multiple spatial and temporal scales (e.g., treefall gaps, fire, wind) impede competitive exclusion (Vandermeer et al. 1996, Uriarte et al. 2009), and where dispersal limitation, chance, and history act together to promote

Manuscript received 21 December 2010; revised 3 May 2011; accepted 14 July 2011. Corresponding Editor: L. Gough.

5E-mail: mu2126@columbia.edu high species richness (Hubbell and Foster 1986). Mechanisms that have the potential to promote species coexistence in the context of repeated disturbance include life history trade-offs between species in colonization and competitive ability (i.e., the competition-colonization hypothesis; Tilman 1994) or in the ability to survive at low resource conditions (e.g., low light in the forest understory) and to exploit the temporary resource-rich conditions (e.g., higher light in the understory) often generated in the wake of disturbance (i.e., the successional niche hypothesis, sensu Pacala and Rees 1998). A trade-off between susceptibility to disturbance and responses to postdisturbance resource-rich conditions may also contrib- 
ute to the maintenance of species diversity in forests (Zimmerman et al. 1994, Loehle 2000).

Understanding the importance of these trade-offs in maintaining species composition in tree communities requires estimates of the relative contribution of several demographic mechanisms that may contribute to successional diversity in the presence of periodic disturbance. First, both the disturbance itself and the high resource conditions generated in its wake may alter juvenile and adult tree growth and survival (Uriarte et al. 2004, Comita et al. 2009). Tree species' ability to produce new sprouts and branches in response to damage and higher resource availability may also impact growth and survival (Putz and Brokaw 1989, Zimmerman et al. 1994, Bellingham 2000). Second, fecundity of reproductive trees may be affected by individual tree damage and by subsequent changes in resource availability. Quantifying this effect requires observations of species-specific variation in fecundity schedules as the community recovers from disturbance. Third, enhanced resource conditions after disturbance may influence rates of seedling establishment. Finally, shade tolerance, the ability of tree species to tolerate the low light resource conditions between disturbance events, is a critical aspect of understanding life history strategies in communities subject to periodic disturbance, because quiescent periods between individual disturbance events offer a window of time in which some species may reach reproductive size classes and therefore remain an important component of the community (Clark 1996, Uriarte et al. 2009). As such, shade tolerance must be considered in any investigation of the role of life history trade-offs in mediating successional dynamics (Pacala et al. 1996).

In order to assess the role of life history trade-offs among species in fostering diversity in tree communities subject to periodic disturbance, we must first define and quantify two key aspects of species-specific responses to disturbance: susceptibility to damage and subsequent survival and recovery in terms of growth and reproductive output. Species' susceptibility to damage includes not only the probability of being damaged, but also the immediate demographic consequences of damage for individual trees, in terms of reduced growth, survival, or fecundity. Thus, susceptibility refers to the immediate negative consequences of being damaged adjusted by the probability of damage. In contrast, recovery refers to changes in demographic rates from pre-disturbance to post-disturbance conditions. Recovery occurs over a longer period, namely the time over which resources establish a clear trajectory toward pre-disturbance conditions. As such, recovery must incorporate repair of damage as well as the potential for increased diameter growth, survival, or reproduction via seeds or sprouting in response to the high resource conditions (e.g., elevated understory light levels) that often follow disturbance events. Recovery must also include the potential for species to colonize sites that become available as a result of disturbance (i.e., canopy gaps created by treefalls).

In this paper, we employ a hierarchical Bayes model to examine species-specific variation in responses to disturbance in the wake of two major hurricanes in a tropical tree community in Puerto Rico. We also investigate whether species exhibit the life history trade-offs that may allow them to persist in the context of repeated disturbance. To this end, we use demographic census data for 10 tree species collected in four censuses of a permanent 16-ha mapped Luquillo Forest Dynamics Plot (LFDP) taken at approximately 5-year intervals, together with annual seed trap data collected over 13 years (1992 through 2005) in the same site, and assessments of damage after each of the two hurricanes. The 10 species represent a range of life history strategies, successional stages, and responses to hurricane damage (Table 1; see also Uriarte et al. 2009). Specifically we ask the following questions:

(1) How do tree species vary in growth, fecundity, and survival responses to hurricane damage (susceptibility) and to the high resource (low crowding and high understory light) conditions generated in the wake of a hurricane (recovery). Our expectation is that species strategies in response to disturbance will vary widely in susceptibility and recovery, and that species with low susceptibility to hurricane damage will recover quickly from damage. In other words, we predict a negative correlation between susceptibility and recovery. We also expect that recovery strategies will depend on the degree to which species rely on sprouting, repair of adult damage, increased growth and survival of juvenile and adult trees in response to enhanced resource availability after storms, or long-distance seed dispersal (colonization) as recovery mechanisms.

(2) Are there life history trade-offs among species in their ability to tolerate the low light conditions between hurricane disturbance events and recovery potential after a hurricane? We expect that shade-tolerant species that typically suffer little damage during a storm will redirect few resources to repair and increased growth, whereas species that suffer significant damage (shade intolerant) will have a strategy that either ensures rapid repair of damage with reduced seed output as resources are directed to repair the damage, or increases seed output to enable population recovery via recruitment of new individuals. These responses may be mediated by competition-colonization trade-offs (low shade tolerance and long-distance colonization) or by successional niche processes (high shade tolerance and recovery potential).

\section{Methods}

Study area and species selection.-The Luquillo Forest Dynamics Plot (LFDP) is a 16-ha forest plot (southwest corner $18^{\circ} 20^{\prime} \mathrm{N}, 65^{\circ} 49^{\prime} \mathrm{W}$ ) located in the Luquillo Mountains of northeastern Puerto Rico. The plot is 500 $\mathrm{m} \mathrm{N}-\mathrm{S}$ and $320 \mathrm{~m} \mathrm{E}-\mathrm{W}$. The forest is classified as 
TABLE 1. Seed mass, sexual system, and successional status of 10 selected species at the Luquillo Forest Dynamics Plot (LFPD), Puerto Rico.

\begin{tabular}{lllllrr}
\hline \hline \multicolumn{1}{c}{ Species (and family) } & \multicolumn{1}{c}{ Code } & $\begin{array}{c}\text { Seed } \\
\text { mass }(\mathrm{g})\end{array}$ & $\begin{array}{l}\text { Sexual } \\
\text { system }\end{array}$ & $\begin{array}{c}\text { Successional } \\
\text { status }\end{array}$ & $\begin{array}{c}\text { Mean and } \\
\text { (maximum) size (cm) }\end{array}$ & $\begin{array}{c}\text { Sapling } \\
\text { mortality }(\%)\end{array}$ \\
\hline Alchorneopsis floribunda (Euphorbiaceae) & ALCFLO & 0.007 & dioecious & pioneer & $7.57(48.99)$ & 35.2 \\
Casearia arborea (Flacourtiaceae) & CASARB & 0.05 & bisexual & secondary & $4.42(51.70)$ & 26.83 \\
Casearia sylvestris (Flacourtiaceae) & CASSYL & 0.006 & bisexual & secondary & $3.61(27.08)$ & 16.4 \\
Dacryodes excelsa (Burseraceae) & DACEXC & 1.24 & dioecious & late & $19.65(85.25)$ & 8.68 \\
Guarea guidonia (Meliaceae) & GUAGUI & 0.4 & bisexual & late & $12.21(104.7)$ & 37.89 \\
Homalium racemosum (Flacourtiaceae) & HOMRAC & 0.011 & bisexual & late & $17.85(124.7)$ & 15.85 \\
Laetia procera (Flacourtiaceae) & LAEPRO & 0.007 & bisexual & late & $6.54(51.59)$ & 10.14 \\
Manilkara bidentata (Sapotaceae) & MANBID & 0.594 & bisexual & late & $9.92(85.36)$ & 6.32 \\
Schefflera morototoni (Araliaceae) & SCHMOR & 0.03 & bisexual & pioneer & $4.06(68.32)$ & 40.48 \\
Tabebuia heterophylla (Bignoniaceae) & TABHET & 0.01 & bisexual & secondary & $9.19(70.38)$ & 21.07 \\
\hline
\end{tabular}

Notes: To determine successional status, we used the primary forest species definition from Smith (1970) and additional studies (Devoe 1989, Lugo and Zimmerman 2002) that combine species-specific characteristics of seed size, seedling regeneration under different light conditions, and relative densities of various life history stages in this forest type. Species were classified as pioneers if their seeds germinate only in gaps where full sunlight arrives at ground level for at least part of the day; these species have high sapling mortality and low seed mass in the shade. Secondary-forest species are species dominating forests that develop after human disturbance; they generally have high sapling/adult ratios and low seed mass but can germinate in partial shade. Late-successional species are those that dominate forests undisturbed by humans; they tend to have large seed sizes (low dispersal ability) and high shade tolerance, and they can reach large diameters. Here, Guarea guidonia appears to be an outlier. We classified it as latesuccessional because it persists in mature forests where other late-successional species dominate. Dry seed mass was from J. ForeroMontaña (unpublished data).

subtropical wet in the Holdridge life zone system. Elevation ranges from $333 \mathrm{~m}$ to $428 \mathrm{~m}$ a.s.l. All of the soils are formed from volcaniclastic sandstone. The LFDP contains 89 species of trees with stems $\geq 10 \mathrm{~cm}$ dbh (diameter at breast height) distributed across 72 genera and 38 families (Thompson et al. 2002). Fortyfive of these species are rare $(<1 \mathrm{stem} / \mathrm{ha}$ of $\mathrm{dbh} \geq 10$ $\mathrm{cm}$ ) and over $75 \%$ of species have fewer than 5 stems/ha. We limited our analyses to a group of 10 tree species that represent a broad range of life history strategies (Table 1).

Tree censuses and hurricane damage.-Hurricane Hugo, a category 4 tropical storm, struck the forest in September 1989, causing significant damage (Zimmerman et al. 1994). The first LFDP census started in 1990 and was completed in 1993. The initial survey recorded several qualitative and quantitative observations on tree damage resulting from the hurricane. For these analyses, damage observations were classified into three categories to represent the degree of hurricane injury for each stem with $\mathrm{dbh} \geq 10 \mathrm{~cm}$ : (1) no damage or light damage ( $\leq 25 \%$ of crown volume removed by the storm), (2) medium damage (25-75\% of crown volume lost through a combination of branch damage and crown break), or (3) heavy or complete damage ( $>75 \%$ of the crown lost, stem snapped, root break or tip-up); see Uriarte et al. (2004). Many damaged trees sprout and contribute significantly to the structure of the forest. In the censuses, one stem (usually the largest diameter) on a multiple-stemmed plant is designated as the main stem. All sprouts that arise either along the whole length of a prone stem or from the base of the stem and also have $\mathrm{dbh} \geq 1 \mathrm{~cm}$ are tagged and measured and associated with the main stem.

The second LFDP census was carried out between November 1994 and October 1996 and included all live stems with $\mathrm{dbh} \geq 1 \mathrm{~cm}$, together with the status (dead or alive) of all stems tagged in the first census. The third and fourth censuses were completed in 2000-2001 and 2005-2006, respectively. As the forest was recovering from Hurricane Hugo, Hurricane Georges struck Puerto Rico in September 1998 with winds up to $150 \mathrm{~km} / \mathrm{h}$ (category 3). Georges severely damaged $16 \%$ and defoliated $27 \%$ of adult trees in the LFDP (J. Thompson and J. K. Zimmerman, unpublished data). Between November 1998 and April 1999, we selected 40 points in a regular $60 \times 60 \mathrm{~m}$ grid across the LFDP. In a $30 \times$ $30 \mathrm{~m}$ square around each of these center points, we assessed the degree of hurricane damage to all trees with $\mathrm{dbh} \geq 10 \mathrm{~cm}$ using methods similar to those described for Hurricane Hugo.

Seed counts.-Seeds were collected from 120 traps placed at every $8 \mathrm{~m}$ of established trails at a distance $8 \mathrm{~m}$ perpendicular to the trail (Appendix A: Fig. A1). The traps had surface area of $0.16 \mathrm{~m}^{2}$ and were constructed using $1-\mathrm{mm}$ mesh mounted $80-100 \mathrm{~cm}$ above the ground. Data analyzed here were counts of seeds collected biweekly (once every two weeks) beginning in 1 April 1992 and continuing through the end of December 2005. To ensure sufficient data for robust parameter estimation, our analyses were restricted to those species having seeds that were found in at least 150 trap collections during the 13-year sampling period. For modeling purposes, we summed the number of seeds of each species collected in each trap over a year as a measure of annual seed input per species at the site.

\section{Hierarchical Bayes Models of Growth, Survival, AND FECUNDITY}

The adoption of a hierarchical Bayes (HB) framework allows us to coherently combine demographic data collected at different temporal (i.e., 5-year interval vs. 
biweekly) and spatial (i.e., individual tree, seed trap) scales and from different ontogenetic stages (e.g., seed, tree) to identify relationships among life history schedules and how they vary across species (Fig. 1, Table 2). HB models can also accommodate multiple sources of variation and uncertainty at different scales. This approach allows us to capture how interactions at one scale, say variation among individuals in response to resource availability, or in these same responses within individuals across years, relate to processes and observations at another, such as variation between species in responses to changing resource environments. This is a critical issue in understanding variability and constraints in the expression of life history trade-offs because species do not respond as an aggregate unit to changing resources, rather individuals do. Using an $\mathrm{HB}$ framework allows us to aggregate individual tree responses to the species scale while incorporating variation among individuals and across time.

The HB approach also allows us to model growth, fecundity, and mortality risk of each tree as latent states that are not directly observed but are rather inferred (through a statistical model) from measured data. These latent demographic states respond to variables that are observed in several ways. There is both individual (e.g., size, wind damage) and population-level variation in demographic rates between years (e.g., year effects on demographic rates); see Fig. 1. Each of these latent states is influenced not only by covariates but also by process misspecification: the extent to which the model structure fails to capture existing relationships between relevant environmental covariates and demographic rates. Because information enters the model in many ways, there is substantial correlation among observations, which can be properly integrated if we allow that the underlying process is stochastic. For instance, fecundity estimates are informed by seed trap data, observations of maturation status, tree size, and hurricane damage, as well as interannual variability (Fig. 1). Exploiting the conditional independence of each data set, together with the underlying process stochasticity, allows us to marginalize across data sets and, thus, draw proper inference on demographic rates (Clark et al. 2010). Here we provide a brief summary of the model, with emphasis on the responses to hurricane disturbance. Detailed descriptions of the modeling approach and computation are provided in Clark et al. (2010). A list of parameters included in the model presented here is provided in Appendix B.

Gender and maturation.-The gender of a tree remains constant, whereas maturation status changes from immature to mature depending on covariates. Maturation was treated as a binary response (mature/immature). Because the transition from immature to mature cannot be observed, it was treated as a hidden Markov process (Clark et al. 2004). Probability of maturation $(\theta)$ increases with tree size and with favorable resource conditions. The probability of maturation for tree $i$ at time $t$ is estimated using a logit link to diameter $(D)$ and the inverse of crowding $(-\ln [1 / \mathrm{NCI}])$ :

$$
\operatorname{logit}\left(\theta_{i, t}\right)=\beta_{0}^{\theta}+\beta_{1}^{\theta} D_{i, t}-\beta_{2}^{\theta} \ln (\mathrm{NCI})_{i, t} .
$$

The Neighborhood Crowding Index (NCI) accounts for neighbor crowding effects on growth and fecundity, a measure of resource availability (Uriarte et al. 2004). For tree $i$ with $m$ neighbors within a $15 \mathrm{~m}$ radius, NCI takes the following form:

$$
\mathrm{NCI}_{\text {focal }, m}=\sum_{i=1}^{n_{m}}\left(\mathrm{DBH}_{i}\right)^{2} /\left(\text { distance }_{i}\right)^{2} .
$$

The formulation in Eq. 1 assumes that probability of maturation increases with the inverse of NCI (i.e., lower crowding increases resources, potentially leading to higher probability of reaching mature status). The choice of a $15-\mathrm{m}$ neighborhood was based on previous analyses about the scale at which neighborhood interactions influence tree growth and mortality (Uriarte et al. 2004). NCI for each individual was calculated using trees at the closest census date.

Maturation status and gender identification for the dioecious species Dacryodes excelsa were determined by repeated visits to all trees in the plot with $\mathrm{dbh} \geq 10 \mathrm{~cm}$ over the course of two years (2006-2007) (ForeroMontaña et al. 2010). For D. excelsa, the unconditional probability that individual $i$ is female is termed the female fraction $\operatorname{Pr}\left(H_{i}=1\right)=\phi$ for observations in any year $t$. The species Alchorneopsis floribunda is also dioecious but no information on the sex of individuals is available. For this species, and for D. excelsa individuals for which gender was unavailable for some years, information enters solely though seed rain data. If seed density near an individual is high, then the probability that it is female is large, and vice versa. See Clark et al. (2004, 2010) for details.

Seed rain and fecundity.-Fecundity (annual seed production per individual) can rarely be observed directly in forest canopies. However, we can infer fecundity from seed trap data that are linked to individual trees via a dispersal model (Clark et al. 2010). Seed counts for each species in each trap depend on the tree location relative to seed traps as well as the tree size, maturation status, gender, and previous hurricane damage of potential parent trees (Fig. 1). For each species, the vector of expected seed densities $\left(s_{t}\right)$ in seed traps 1 through $k$ in year $t$ is

$$
s_{t}=\mathbf{T}\left(c \mathrm{BA}+\mathbf{K}_{t} f_{t}\right)
$$

where $\mathbf{T}$ is the vector of seed trap areas, BA is the basal area of the species in the plot, $c$ is a fitted parameter, $\mathbf{K}_{t}$ is the $k$ by $n_{t}$ matrix of dispersal kernel values, and $f_{t}$ is the length of the $\mathbf{n}_{t}$ vector of fecundities that includes all fecund trees in the plot for that year. The first term allows for seed inputs from outside the mapped plot and the second term is a measure of source strength. The dispersal kernel $(K)$ is assumed to 


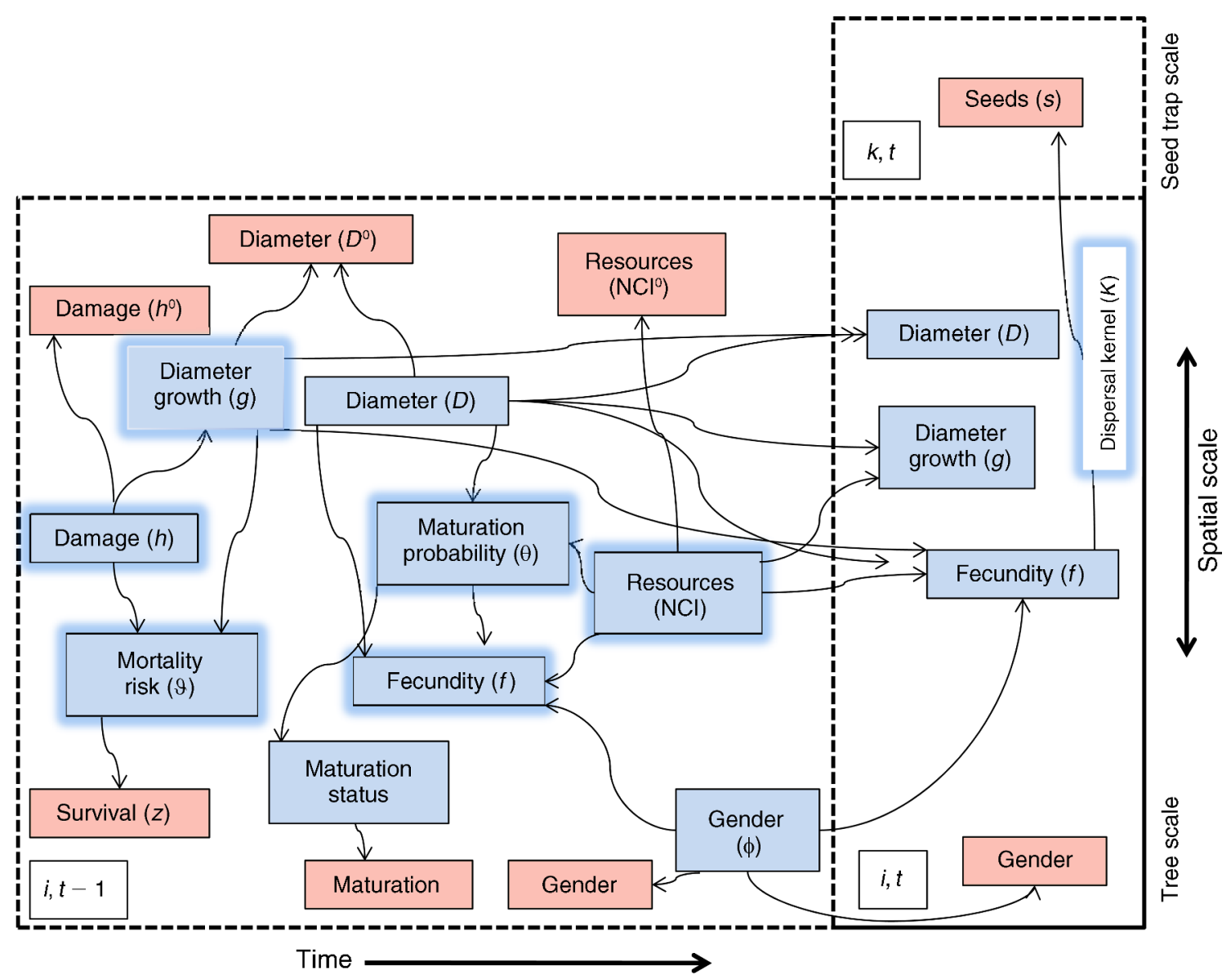

FIG. 1. A simplified directed acyclic graph of interactions and data for tree $i$ in year $t$. Latent states are blue and include annual estimates of diameter $(D)$, diameter growth $(g)$, hurricane damage $(h)$, maturation probability $(\theta)$, maturation status $(Q)$, fecundity $(f)$, gender, mortality risk $(\vartheta)$, and resource availability $(\mathrm{NCI})$. Latent states are not directly observed, but are rather inferred (through the statistical model) from other variables that are directly measured (data). Data are shown in pink and include 5-year diameter measurements $D^{0}$, 5-year survival observations, hurricane damage $h^{0}$, gender observations, and our index of resources, $\mathrm{NCI}^{0}$, also measured at 5-year intervals (see Table 2). Seed data $(s)$ are collected in seed traps $(k)$ at the $0.5-\mathrm{m}^{2}$ scale rather than at the tree scale. Fecundity for tree $i$ in year $t$ is informed by seed counts collected in year $t$ in the subset of the 120 seed baskets that fall within the estimated seed dispersal kernel $(K)$ of tree $i$. The seed dispersal kernel is estimated at the species scale but takes into account differences in fecundity between individual trees within a species and provides an estimate of maturation status. Successional niche processes will lead to trade-offs between high shade tolerance and recovery potential. Tolerance is defined by mortality risk, diameter growth, and fecundity responses to low resource $(-\ln [\mathrm{NCI}])$ conditions. Recovery is defined by the change in individual tree diameter growth, mortality risk, or fecundity between low and high resource conditions. Relevant demographic parameters for this trade-off are highlighted with a blue halo. Colonization-competition trade-offs will be determined by the response of individual tree mortality risk, diameter growth, and fecundity to low resources (boxes with halos) and the ability of species to colonize new sites, which is determined by fecundity and the estimated seed dispersal kernel, estimated from seed counts and locations.

follow a two-dimensional Student's $t$ distribution, with scale parameter $u$ (Clark et al. 2010). Seed count data follow a Poisson distribution. In addition to being informed by seed trap data, fecundity also depends on tree size and hurricane damage. The relationship between size, damage, and fecundity is captured by a bivariate state-space model for diameter growth and fecundity.

TABLE 2. Summary of data used in the model.

\begin{tabular}{|c|c|c|c|c|}
\hline Data & Time span & Spatial extent & $\begin{array}{c}\text { Frequency } \\
\text { of sampling }\end{array}$ & Data collected \\
\hline Trees with $\mathrm{dbh}>1 \mathrm{cn}$ & $1990-2005$ & 16 ha & 5 years & diameter, location, status \\
\hline Damage to trees with $\mathrm{dbh}>10 \mathrm{~cm}$ & 1990 & 16 ha & NA & tree damage, Hurricane Hugo (1990) \\
\hline Damage to trees with $\mathrm{dbh}>10 \mathrm{~cm}$ & 1998 & $4030 \times 30 \mathrm{~m}$ plots & NA & tree damage, Hurricane Georges (1998) \\
\hline Seeds & $1992-2005$ & $1200.5-\mathrm{m}^{2}$ traps & biweekly & counts \\
\hline
\end{tabular}


Diameter growth and fecundity.-Diameter growth is informed by census data collected at 5-year intervals, by observations of damage collected after Hurricanes Hugo and Georges, and by a state-space model that includes fecundity. Because census data were collected at 5-year intervals, we modeled diameter growth using a two-step process. First, we used census data to model means and standard deviations for diameter and diameter increments for each tree-year (J. S. Clark et al. 2007), recognizing that there is no individual year information beyond the interpolation bounded by 5 -year observations. When information is missing, e.g., for diameter in year $t$, then the model for year $t$ is parameterized using data augmentation (Elerian et al. 2001). Data augmentation refers to one of several numerical devices to deal with missing information. In our case, the basic timescale of inference is annual, so augmentation for missing census years is a sensible approach. As is often the case, it is implemented here as an efficient computational device and not for purposes of drawing inference on annual fluctuations in growth. In other words, we are modeling growth at the annual scale, but focusing inference at multiyear scales. Uncertainty associated with this interpolation is described by posterior estimates of diameter growth (J. S. Clark et al. 2007). These posterior estimates are then used as priors in the second step following the approach outlined in Clark et al. (2010). Seed trap data are available at the annual scale so no data augmentation is required to model fecundity.

The second step of the analyses is a state-space model for growth and fecundity. Once trees are mature, fecundity depends on covariates, including size, hurricane damage, and neighborhood crowding (Eq. 2) as covariates that are likely to affect demography, together with individual and year effects. Year effects reflect annual variation in demographic rates that are shared by the whole population. These effects capture some level of demographic synchronicity among individuals due to masting and climate variation. There are also individual differences beyond those accounted for by covariates. Individual random effects allow us to account for genetic and environmental variation (e.g., soil water or nutrient status) not included in the model. There is error in the model itself. Because both diameter growth and fecundity are response variables, we include them together as a multivariate regression within the statespace model. This approach allows us to explicitly include error covariance between growth and fecundity beyond that which is accommodated by covariates. The growth-fecundity state model is

$$
\mathbf{y}_{i, t}=\mathbf{x}_{i, t-1} \mathbf{A}+b_{t}+r_{i}+\varepsilon_{i, t}
$$

where the response vector $\mathbf{y}_{i, t}$ for tree $i$ in year $t$ includes both growth and fecundity $(2 \times 1$ dimension $), b_{t}$ are year effects, $r_{i}$ are random individual effects, and $\varepsilon_{i, t}$ is the error term. $\mathbf{A}$ is the matrix of estimated parameters with dimensions $2 x p$, where $p$ is the number of covariates included in the growth-fecundity state-space model. The vector $\mathbf{x}_{i, t-1}$ includes all the fixed covariates: hurricane damage $(h)$, a categorical variable that can take three values (see Methods: Tree censuses and hurricane damage), diameter $(D)$, diameter growth $(g)$ in the previous time interval, and an index of neighborhood crowding (NCI), taking the following form:

$$
\begin{aligned}
& \mathbf{x}_{i, t-1}= \\
& {\left[\begin{array}{lllll}
1 & h_{i, t-1} \ln \left(D_{i, t-1}\right) & \ln \left(D_{i, t-1}\right)^{2} & -\ln (\mathrm{NCI}) & \ln \left(g_{i, t-1}\right)
\end{array}\right] .}
\end{aligned}
$$

Previous studies have demonstrated that trees can regrow branches and repair damaged crowns within relatively short time periods of 3-4 years (e.g., Walker 1991), so trees that were recorded as damaged in this study were assumed to recover to a healthy status within a 4-year period. However, in that 4-year period, damage may have affected tree diameter growth and fecundity (Uriarte et al. 2004). The effects of damage on growth and fecundity may not necessarily change monotonically during the recovery period because trade-offs between diameter and fecundity vary together with shifts in resource availability (e.g., changes in light availability) that may alter tree demography in unexpected ways. For this reason, the effects of damage were modeled with a nonparametric approach, using a $4 \times 1$ multinomial vector ( $h$ in Eq. 5) for both medium and complete damage categories (see Field methods). This formulation allows for a 4-year recovery period, in line with field observations (Walker 1991).

The $\ln (D)$ term in Eq. 5 accounts for increases in fecundity and growth with size, whereas the $\ln (D)^{2}$ captures senescence effects. The growth term $g_{i, t-1}$ accounts for potential trade-offs between growth and fecundity across years. Again, although annual estimates of growth are available in the form of posterior distribution, we interpret the posteriors aggregated over 4-year intervals, the hypothesized period of recovery from hurricane damage. This formulation assumes that growth and fecundity increase with the inverse of NCI $[-\ln (\mathrm{NCI})]$, our index of neighborhood crowding (Eq. 2).

Growth and fecundity conditionally depend on several sources of data. Fecundity depends on both seed and tree data. The growth model depends on prior mean growth calculated in the first step of the analyses, fixed covariates for growth (hurricane damage, size, diameter growth in the previous time interval, and neighborhood crowding), seed data, and survival probability. An additional model for growth was fitted using all juvenile trees. The model is univariate but includes the same covariates as the adult model. Details are provided in Clark et al. (2010).

Survival.-To avoid unrealistic assumptions about the functional form relating growth rate to survival, we used a nonparametric approach that combines the effects of diameter growth rate and tree size on survival (Metcalf et al. 2009). In the Metcalf et al. (2009) model, each 
individual each year has two mortality risks, one associated with tree diameter $\left(\mu_{\mathrm{D}}\right)$ because mortality risk can increase with size and age due to physiological decline, and a second one resulting from slow growth $\left(\mu_{\mathrm{g}}\right)$, which is related to overall individual health. Then, the probability that individual $i$ survives from year $t-1$ to $t$ is calculated as

$$
\vartheta_{i j, t}=1-\left(\mu_{\mathrm{g}_{i j, t-1}}+\mu_{\mathrm{D}_{i j, t-1}}-\mu_{\mathrm{g}_{i j, t-1}} \mu_{\mathrm{D}_{i j, t-1}}\right) .
$$

Priors for each bin are determined using a beta density (Appendix B) that is constrained only to represent a monotonically increasing effect for tree size (Metcalf et al. 2009). This approach allows us to separate the distinct effects of growth and size on mortality (Clark et al. 2010).

Prior distributions.- The analysis includes both informative and noninformative prior distributions. In Appendix $\mathrm{C}$ we provide a detailed summary of prior distributions and the rationale for our choices.

Computation and model evaluation.-The posterior distribution was simulated with Metropolis-withinGibbs, based on conditional posteriors detailed in Clark et al. (2010). Computational procedures are described in detail in Clark et al. (2010). A summary of model evaluation results is provided in Appendix D.

\section{Model Interpretation and Results}

To facilitate interpretation of model output, we provide a brief description of the parameters, simulations, and approach used to answer the questions posed in the introduction before presenting the results. (1) How do tree demographic responses to damage (susceptibility) and to the high light conditions generated in the wake of hurricane disturbance (recovery) vary across species? (2) Are there life history trade-offs in the ability of species to tolerate the low light condition between hurricane disturbance events and population recovery potential after a hurricane?

\section{Species variation in susceptibility and recovery following hurricane damage and disturbance}

To assess variation among species in demographic susceptibility to hurricane damage, we compared speciesspecific estimates of the immediate effects of medium and severe damage levels on growth and fecundity in the year immediately following the hurricane (first entry of vector $h$ in Eq. 5). To assess potential effects of damage on survival, we used model output to quantify the effects of (1) diameter growth at a species mean size (dbh), and (2) diameter on maturation probability at a species mean crowding (NCI) levels (Eqs. 1 and 2) on the probability of survival (Eq. 6).

Contrary to our predictions, the immediate responses of species to storm damage were not completely consistent with traditional life history assumptions. For instance, diameter growth for the pioneer Schefflera morototoni did not change significantly in response to severe damage (Fig. 2). Rather, species responses to severe hurricane damage varied, with some exhibiting lower diameter growth following severe damage (e.g., Alchorneopsis floribunda, Casearia arborea, Manilkara bidentata), and others displaying weak or neutral responses (e.g., Laetia procera, Tabebuia heterophylla). Storm damage of medium severity (e.g., loss of branches rather than the whole crown) did not have a substantial effect on diameter growth of any of the species. Regardless of its severity, the posterior densities of hurricane damage on tree fecundity overlapped zero for most species, with only one species (A. floribunda) showing an increase in seed production following damage of medium severity (Fig. 2).

The tendency for diameter growth to decrease in response to hurricane damage indirectly affects probability of survival, because slow growth is associated with higher mortality risk (Fig. 3a). For instance, reductions in average diameter growth in the wake of a severe storm for the late-successional species Dacryodes excelsa and for the secondary-forest species $C$. arborea were similar in magnitude, decreasing $\sim 0.7 \mathrm{~cm}$ after the hurricane (Fig. 2a). However, the effect of a reduction in growth of this magnitude on the probability of survival is substantially greater for C. arborea than for D. excelsa (Fig. 3a). Overall, the probability of mortality for pioneer and secondary-forest species increases steeply below a species-specific diameter growth rate threshold. The range of growth rates at which this threshold occurs does not follow the expectation from traditional life history groupings. For instance, at a relatively high growth rate of $0.5 \mathrm{~cm} / \mathrm{yr}$, probability of mortality is greater for L. procera, a late-successional species, than for C. arborea, a secondary-forest species (Fig. 3a, b). Damaged-induced reductions in diameter growth may also extend the time required for trees to reach reproductive maturity (Fig. 3c,d). These effects would be particularly marked for species that grow slowly and become mature at relatively large diameters, such as $D$. excelsa and M. bidentata.

Species recovery during high resource conditions generated in the wake of a hurricane.-Tree species differ in their recovery from wind disturbance, at the individual tree level through repair of damage and sprouting, and at the population level through juvenile response to enhanced resource availability, seed production, and colonization of new sites. To assess this variation in responses, we estimated recovery of damaged individuals by comparing changes in hurricane effects on growth and fecundity standardized across species from the first to the fourth year following a hurricane (i.e., the first and last values of the $4 \times 1$ multinomial vector $h$ in Eq. 5 using all the trees that fell in each category).

Recovery through seed production and tree growth and survival response to enhanced resource availability was assessed by quantifying changes in demographic rates for all undamaged trees while accounting for variation in crowding conditions between individuals and over time. 


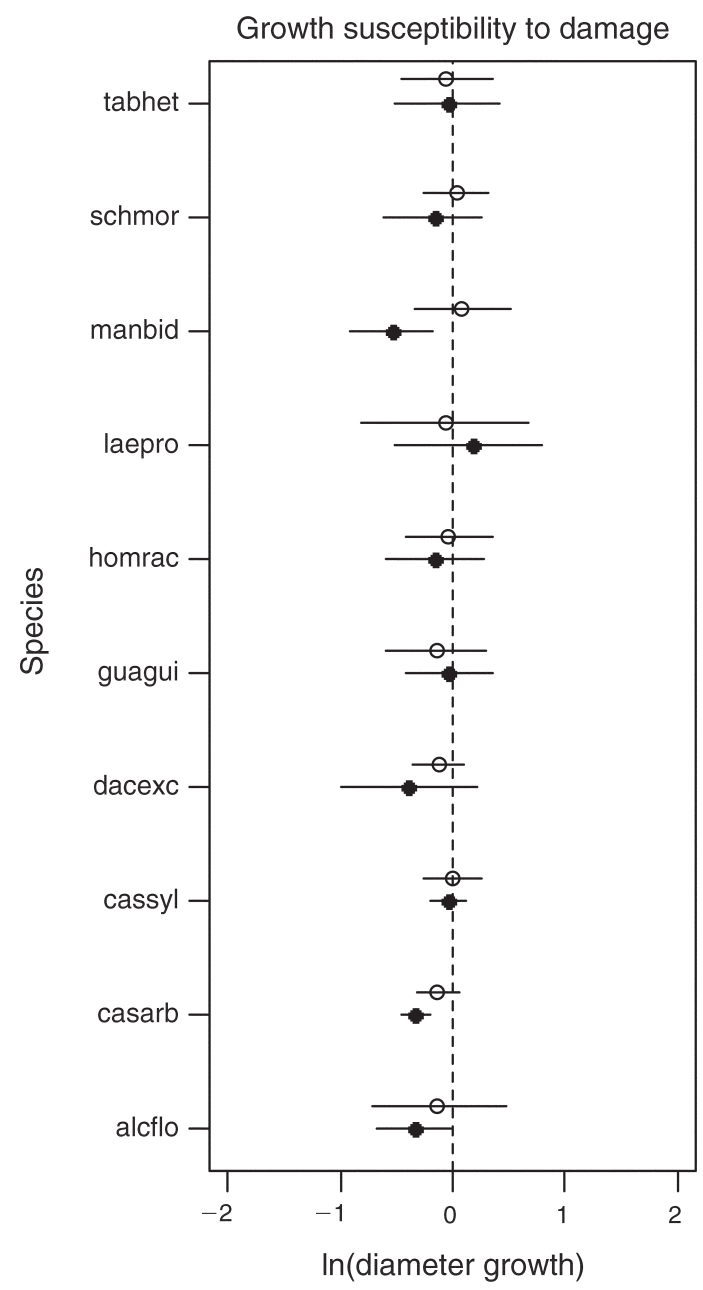

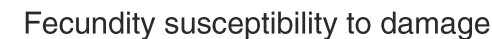

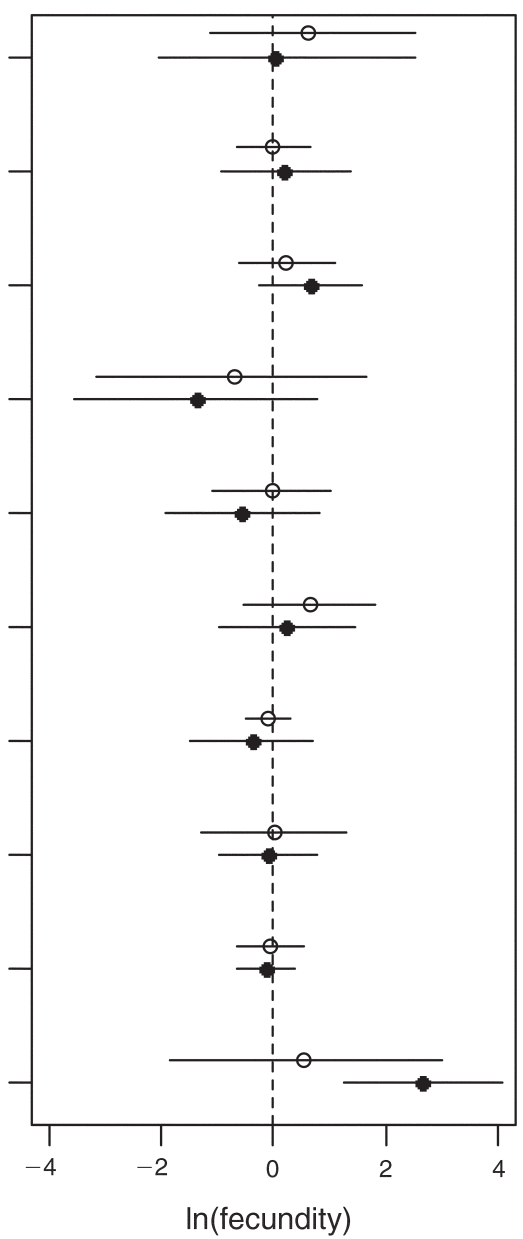

FIG. 2. Susceptibility of species to severe (solid circles) and intermediate (open circles) hurricane damage (see Methods: Tree censuses and hurricane damage for details on damage classification). The ln-transformed values for diameter growth were originally measured in $\mathrm{cm}$, and ln-transformed fecundity estimates were based on seed counts from seed traps. Lines cover 2.5-97.5\% quantiles. Please refer to Table 1 for species abbreviations.

Specifically, we compared demographic rates of individuals with low (0-30\% quantiles) and high (70-100\% quantiles) values of observed crowing conditions. We also used the estimates of individual fecundity (Eq. 5) together with seed dispersal kernels to quantify the potential for long-distance dispersal when new sites become available for colonization after hurricanes open up the canopy. This critical component of population-level recovery was defined here as the fraction of seeds reaching a patch $>50 \mathrm{~m}$ from the source tree. The final component of recovery, sprouting rate, was calculated directly from the census data (Table 3; see Methods: Tree censuses).

Recovery from damage through repair was highly variable across species, ontogenetic stages, and demographic processes. Heavily damaged individuals from the 10 focal species increased fecundity considerably over the 4-year period following a hurricane (Fig. 4; note $\log$ scales). Individuals with heavy damage also exhibited slight decreases in the probability of mortality over the same time period, whereas the recovery of diameter growth was either positive or showed no change. Medium-damaged individuals showed small post-hurricane changes in fecundity and positive increases in diameter growth rate consistent with the slight decrease in probability of mortality.

Responses to enhanced resource availability after storms were evaluated by quantifying demographic rates at high and low crowding conditions (as proxies for low vs. high light availability). When evaluated at the low and high ends of this crowding continuum, species displayed a range of responses (Fig. 5). Interestingly, both pioneers and late-successional species showed stronger responses to favorable, low crowding conditions (i.e., greater growth, fecundity, and survival probability) than secondary-forest species. Overall, demographic responses were greater for adult trees than saplings, with two exceptions: saplings of Guarea guidonia and D. excelsa displayed greater diameter 

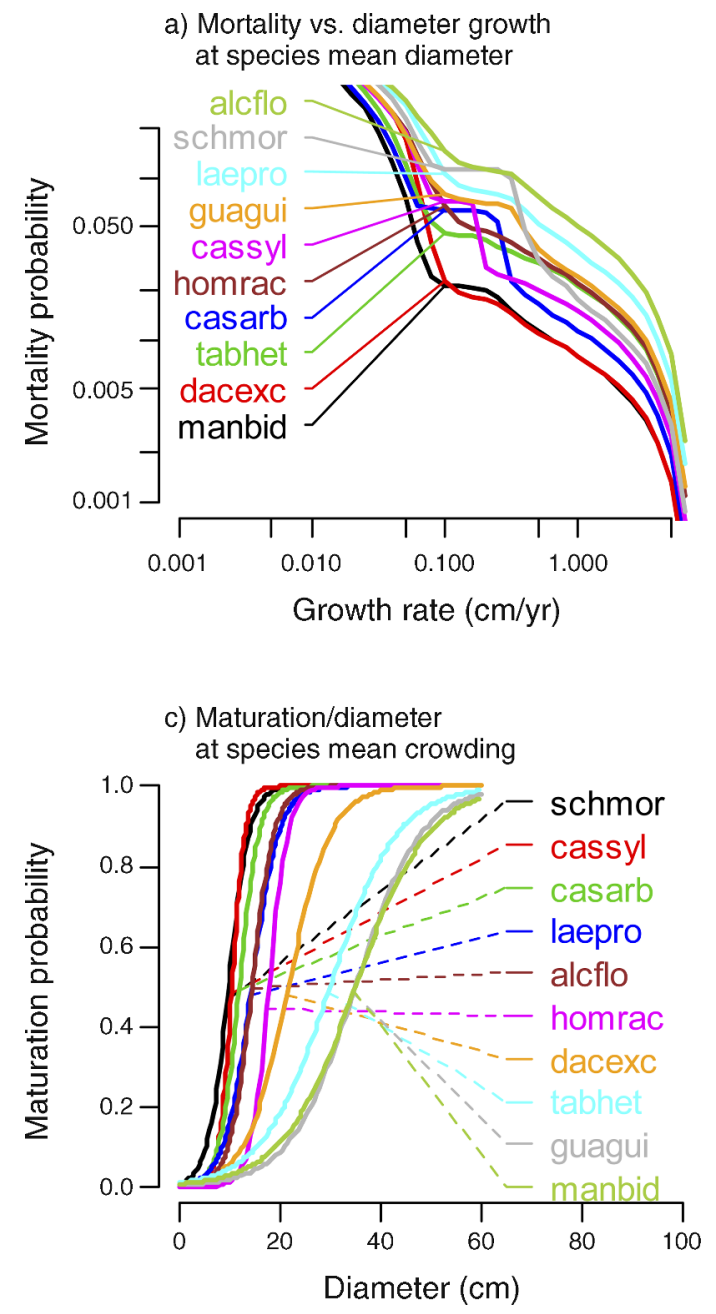

b) Mortality at mean diameter and growth $=0.1 \mathrm{~cm}$

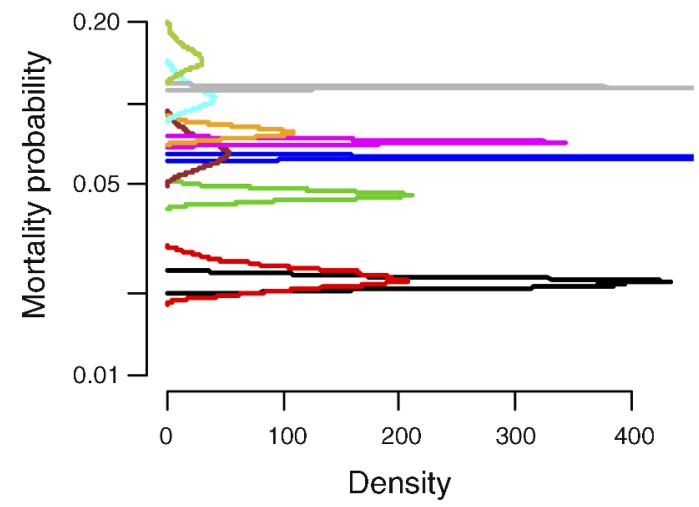

d) Diameter at $50 \%$ maturation probability

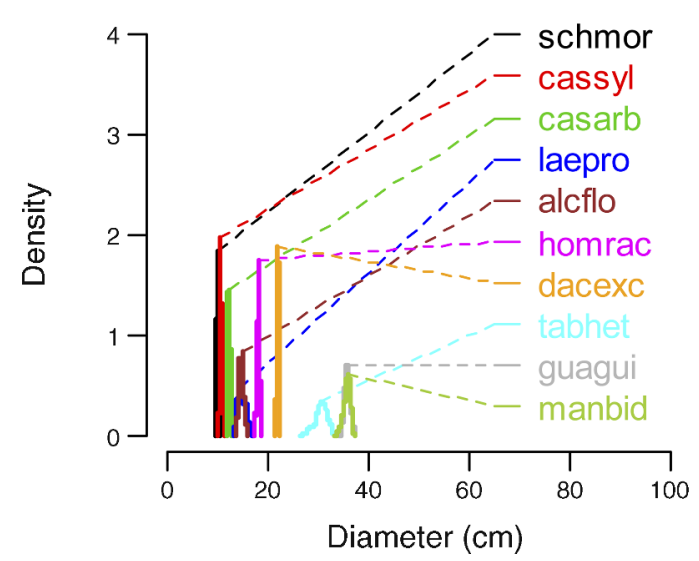

FIG. 3. (a) Posterior mean mortality risk plotted against diameter growth rate. These are nonparametric fits where we assume monotonic priors. (b) Posterior mortality probability distribution (density, not normalized) at diameter growth $=0.1 \mathrm{~cm}$ and species mean diameter. (c) Maturation probability $(\theta)$ as a function of diameter at species mean crowding $(-\ln (\mathrm{NCI}))$ conditions. (d) Diameter distribution (density, not normalized) at which posterior probability of maturation $(\theta)=0.5$. Please refer to Table 1 for species abbreviations.

TABLE 3. Number of stems with $\mathrm{dbh}>1 \mathrm{~cm}$ across the censuses of the Luquillo 16-ha FDP, seed-basket years, and the impact of Hurricane Hugo on the proportion of adult trees damaged and the proportion of stems $(\mathrm{dbh}>1 \mathrm{~cm})$ with basal sprouts.

\begin{tabular}{|c|c|c|c|c|c|}
\hline \multirow[b]{2}{*}{ Species } & \multirow[b]{2}{*}{ No. trees } & \multirow[b]{2}{*}{ No. seed-baskets } & \multicolumn{2}{|c|}{ Proportion of adult trees } & \multirow{2}{*}{$\begin{array}{c}\text { Proportion of stems } \\
\text { with basal sprouts }\end{array}$} \\
\hline & & & Medium damage & Severe damage & \\
\hline Alchorneopsis floribunda & 416 & 262 & 0.18 & 0.38 & 0.22 \\
\hline Casearia arborea & 8385 & 485 & 0.08 & 0.29 & 0.54 \\
\hline Casearia sylvestris & 4193 & 214 & 0.15 & 0.16 & 0.57 \\
\hline Dacryodes excelsa & 1735 & 533 & 0.3 & 0.05 & 0.04 \\
\hline Guarea guidonia & 1034 & 366 & 0.33 & 0.14 & 0.61 \\
\hline Homalium racemosum & 355 & 400 & 0.22 & 0.24 & 0.28 \\
\hline Laetia procera & 344 & 564 & 0.07 & 0.06 & 0.01 \\
\hline Manilkara bidentata & 2330 & 152 & 0.31 & 0.1 & 0.15 \\
\hline Schefflera morototoni & 4605 & 852 & 0.31 & 0.1 & 0.5 \\
\hline Tabebuia heterophylla & 945 & 431 & 0.25 & 0.14 & 0.6 \\
\hline
\end{tabular}

Notes: Seed-basket years are the number of baskets through the study years with nonzero seed counts. Tree damage categories after Hurricane Hugo are defined in Methods. 
a) Fecundity recovery from severe damage

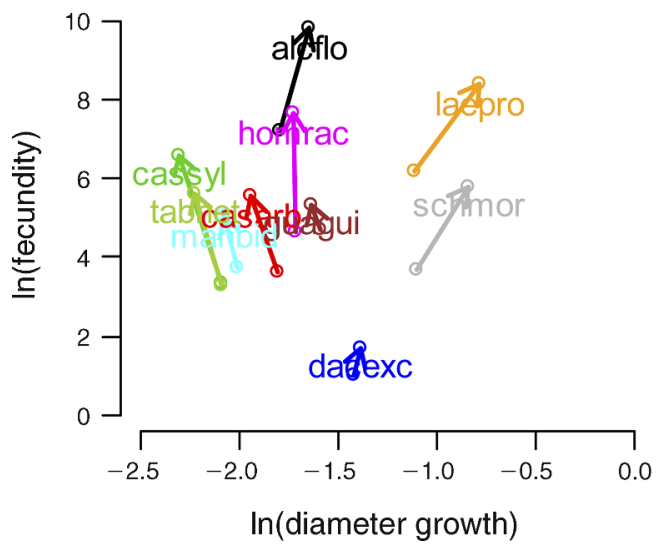

c) Fecundity recovery from medium damage

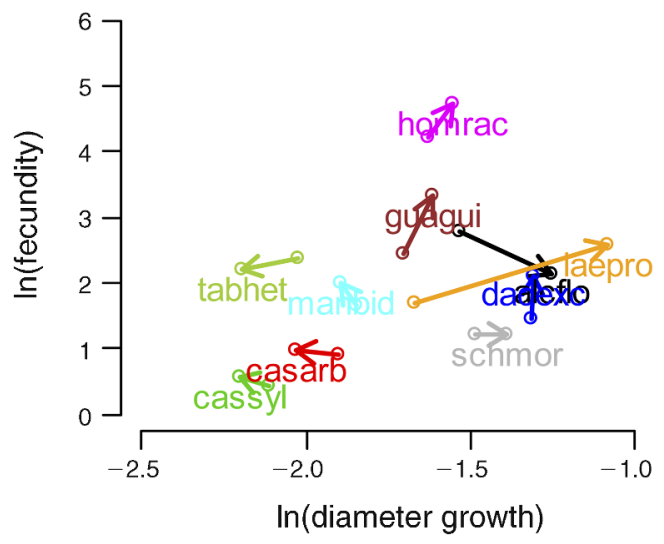

b) Shifts in probability of mortality after severe damage

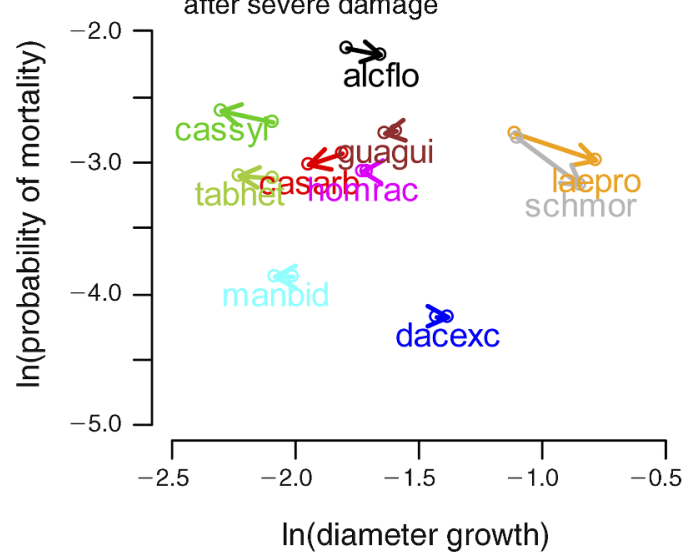

d) Shifts in probability of mortality after medium damage

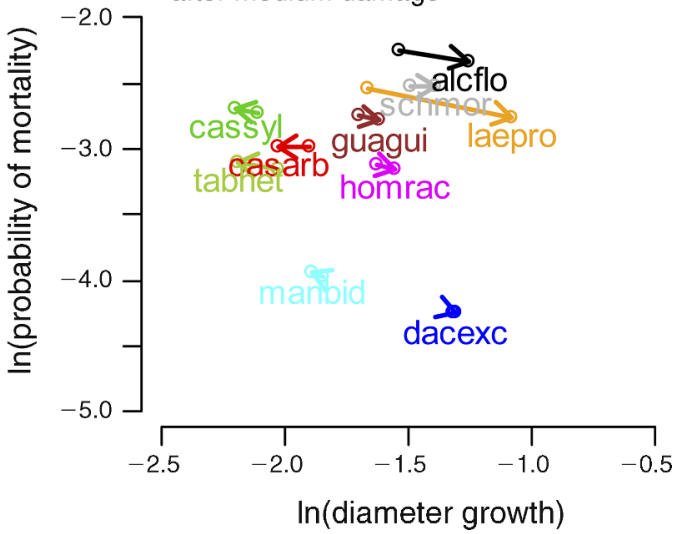

FIG. 4. Recovery response vectors by species to two levels of damage: severe and intermediate (medium). Response vectors were calculated using posterior estimates for the demographic rates of individual that fell within each damage category. Arrows point from time 1 (the year following the hurricane) to time 3 (three years later). Please refer to Table 1 for species abbreviations.

growth responses than adults of the same species (Fig. $5 d$ ). Production of sprouts varied by an order of magnitude among species, with a greater proportion of secondary-forest trees than pioneers and late-successional trees sprouting after Hurricane Hugo (Table 3). Some secondary-forest species such as $T$. heterophylla, however, had a proportion of sprouting individuals comparable to those of some late-successional species (G. guidonia).

We combined estimates of fecundity (Eq. 5) and seed dispersal kernels derived from location of adults and seed traps to quantify species potential for long-distance dispersal as the proportion of seeds dispersed $>50 \mathrm{~m}$ from the source tree. Long-distance colonization ability varied by one order of magnitude among species and showed a moderate negative correlation with seed mass $(r$ $=-0.46, n=10, P=0.08)$. The late-successional species $M$. bidentata had the lowest long-distance colonization ability (proportion of seeds $=0.003$ ) while the wind-dispersed secondary-forest species $T$. heterophylla had the highest (proportion of seeds $=0.032$ ). These proportions did not entirely align with expectations based on life history groupings. For example, long-distance colonization ability for the pioneer S. morototoni was low (proportion of seeds $=0.007$ ) and similar to that of Homalium racemosum, a late-successional canopy dominant.

Our analyses suggest that species find different ways to persist in a hurricane-impacted forest. First, we found a moderate relationship $(r=-0.73, P=0.02)$ between the ability of adult trees to recover from severe damage through repair, as evidenced by a compound metric that incorporates recovery in all demographic parameters (diameter growth, fecundity, and survival; Fig. 5d), and the response of sapling growth and survival to increased resource availability. Second, species exhibited a strong negative correlation between the percentage of stems that initiated basal sprouts in response to hurricane damage and the compound demographic response (i.e., changes in fecundity, growth, and survival) of adults to low crowding conditions (Fig. 6).

Relationship between species damage susceptibility and recovery.-To quantify the relationship between suscep- 
a) Mature fecundity

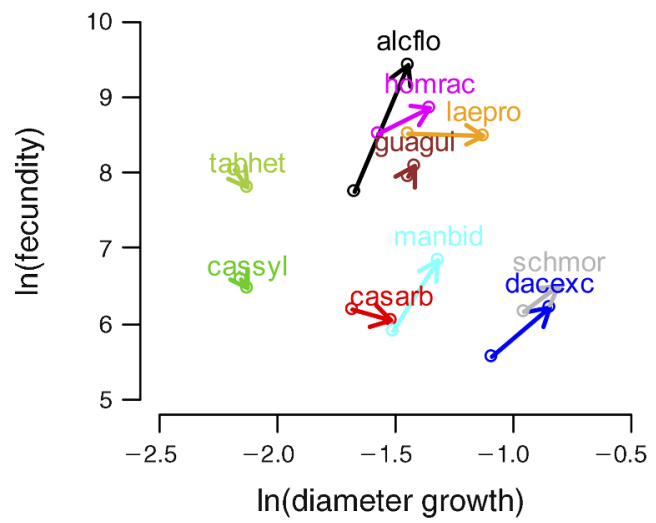

c) Immature probability of mortality

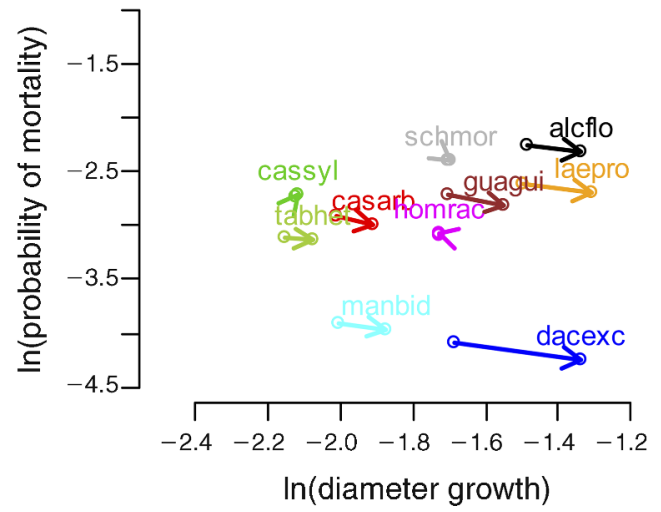

b) Mature probability of mortality

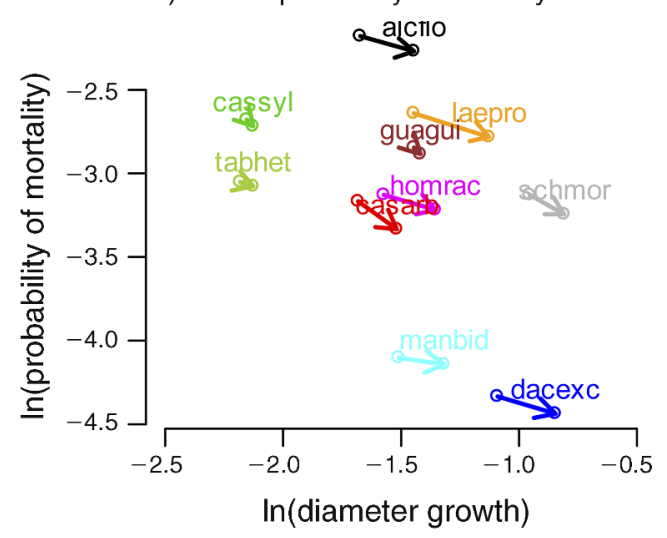

d) Total response

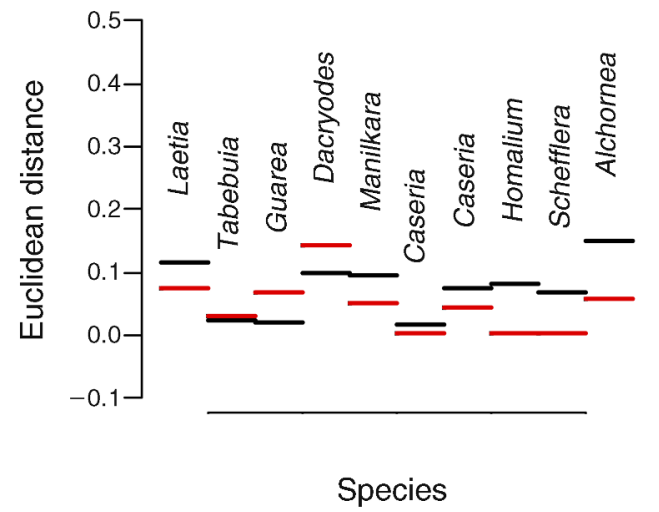

FIG. 5. Resource response vectors by species for two size classes (mature individuals, $40 \mathrm{~cm} \mathrm{dbh}$; immature individuals, $10 \mathrm{~cm}$ $\mathrm{dbh}$ ). Arrows point from demographic rates at high crowding (low resource) conditions to low crowding (high resource) conditions. For panel (d) we calculated multidimensional demographic Euclidian distances standardized to the range of values observed within each species (black, adults; red, juveniles). Please refer to Table 1 for species abbreviations.

tibility to damage during a hurricane and species' ability to exploit the high resource conditions generated after disturbance, we examined correlations between standardized mean posterior values for damage effects on growth, survival, and fecundity and our three metrics of recovery potential: repair of damage, demographic response to changes in crowding as a proxy for resource availability, and long-distance dispersal ability (See Results: Species recovery during high resource conditions). To examine variation in recovery responses while accounting for the probability of damage, we also assessed correlations between the percentage of individuals $(\geq 10 \mathrm{~cm} \mathrm{dbh})$ of each species in each of the two hurricane damage categories, the percentage of individuals that were recorded with sprouts after the hurricane (Table 3), and the recovery metrics.

Contrary to our expectations, we found weak but positive relationships between the immediate hurricane impacts on adult tree growth, survival, and fecundity (susceptibility) and recovery potential. Although these relationships were largely not significant, we found a significant positive relationship between species' reduc- tions in diameter growth rate in the year immediately following hurricane damage (susceptibility), and one of our recovery metrics, namely long-distance dispersal ability $(r=0.64, P=0.04)$. Specifically, species that experienced greater proportional decreases in diameter growth rate in response to damage of medium severity were better long-distance colonizers.

\section{Life history trade-offs in shade tolerance between hurricanes and recovery after a hurricane}

To assess the magnitude of life history trade-offs between species in survival at low resource levels (high crowding) and the ability to exploit the high resource (low crowding) conditions that occur after disturbance (successional niche), we examined correlations between species-specific growth and fecundity at high crowding conditions and posterior mean values for long-distance colonization ability. We also examined correlation between species demographic rates at high and low crowding conditions characterized as before (See Results: Species recovery during high resource conditions). 


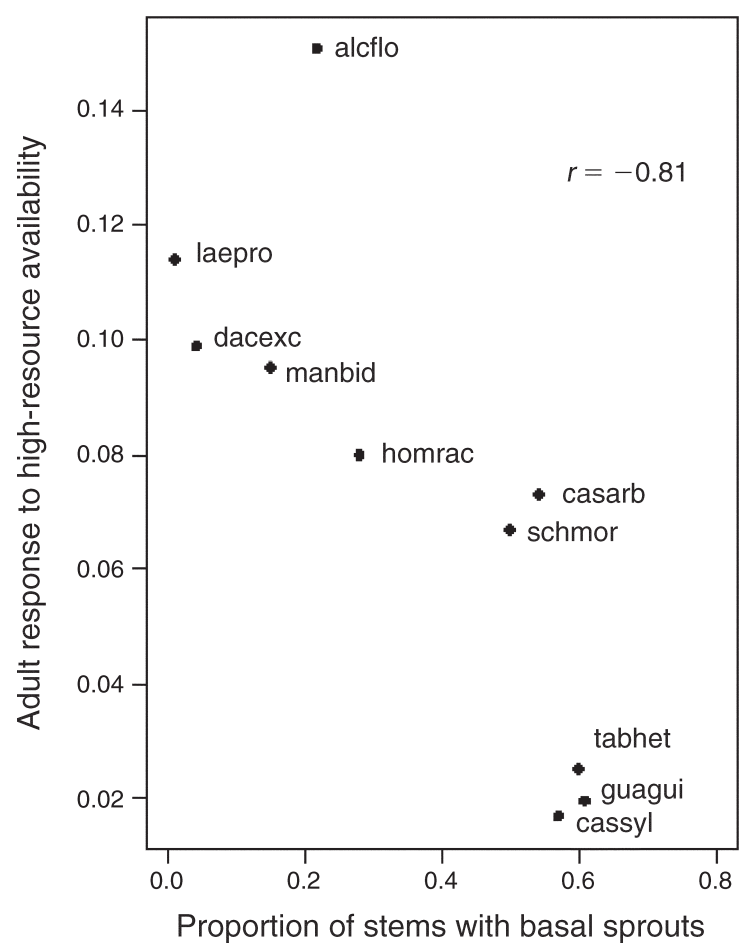

FIG. 6. Relationship between species' sprouting ability (proportion of stems with basal sprouts) and adult response to low crowding conditions. Responses to enhanced resource availability after storms were evaluated by quantifying demographic rates at high and low crowding conditions (as proxies for low vs. high light availability) for adults. See Fig. 5 for details on quantifying the overall adult demographic response, and refer to Table 1 for species abbreviations.

In agreement with expectations from the colonization-competition hypothesis, our analyses showed moderate life history trade-offs in the ability of species to colonize sites $>50 \mathrm{~m}$ from source trees and adult survival at high crowding (low resource) conditions $(r$ $=-0.74, P=0.02 ;$ Fig. $7 \mathrm{a}$ ), in that species with the ability for long-distance colonization had lower survival in high crowding conditions. We also found a trade-off in the ability of species to exploit the temporary resource-rich conditions often generated in the wake of disturbance by increasing seed production (fecundity) and their ability to survive at high crowding conditions $(r=-0.66, P=$ 0.03 ; Fig. $7 b$ ), providing some support for the successional niche hypothesis.

\section{DiscusSION}

Response to disturbance constitutes an important axis of differentiation in tree life histories (Clark 1996, Loehle 2000). We examined variation among 10 tree species in demographic responses to hurricane damage, further partitioning these responses into susceptibility and recovery. By incorporating covariates collected at several temporal and spatial scales and across different ontogenetic stages, our hierarchical Bayes model provides unique insights into the demographic processes and life history trade-offs that mediate tree community responses to disturbance.

\section{Species-specific variation in susceptibility and recovery from hurricane damage}

Susceptibility to damage was relatively low; posterior means for the effects of damage on diameter growth often overlapped zero, especially for individuals that suffered medium levels of damage (e.g., branch loss). Walker (1991) also showed that, even after severe hurricane damage, some tree species in this forest can initiate leaf production as early as two weeks after a storm. Rapid recovery may minimize the effects of hurricane damage on diameter growth. Thus, while species exhibit clear differences in the probability of being damaged during a hurricane (Zimmerman et al. 1994), the consequences of damage appear to be minor, at least in the short term, although reduced growth rate may increase the risk of mortality.

The effects of hurricane damage on fecundity were less apparent than those on diameter growth, and one species (A. floribunda) exhibited a positive effect of hurricane damage on fecundity. This is an unexpected result, given that larger trees, which tend to be more
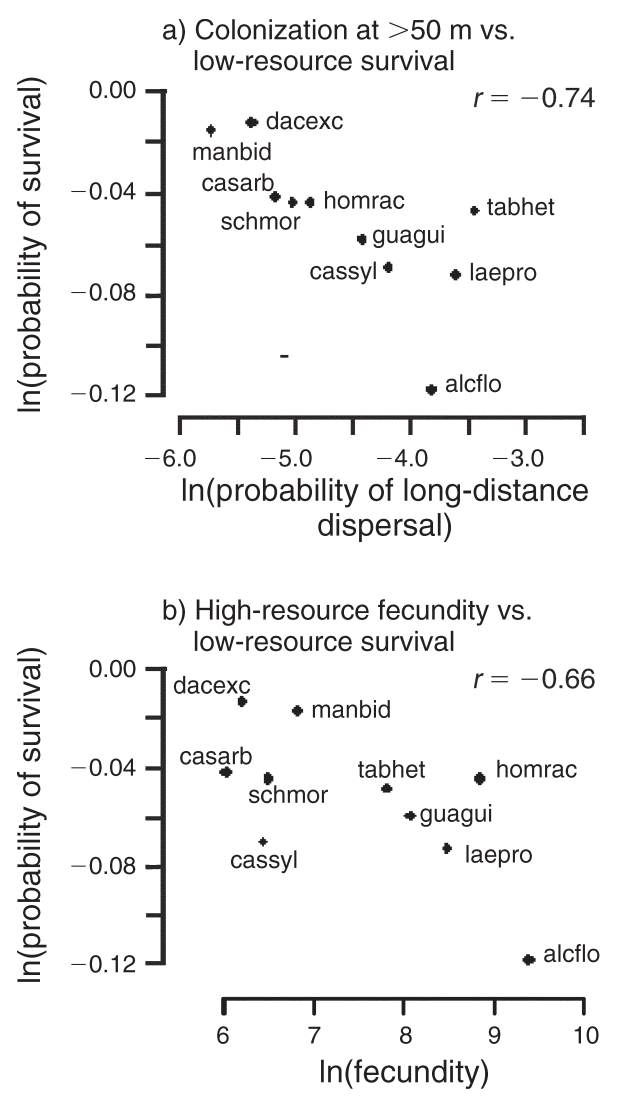

FIG. 7. Trade-offs (a) between low-resource survival and probability of long-distance dispersal and (b) between lowresource survival and high-resource fecundity. Please refer to Table 1 for species abbreviations. 
fecund, are also more likely to suffer hurricane damage (Uriarte et al. 2009). At least two potential reasons may account for this result. First, we started collecting seeds in 1992, well after the Hurricane Hugo struck the plot in 1989. As a result, our analysis primarily captures the effect on fecundity for Hurricane Georges, which was a weaker storm than Hurricane Hugo (Canham et al. 2010). Second, as for diameter growth, rapid recovery may have obscured the initial post hurricane damage responses, particularly after the structural effects of Hurricane Hugo on large trees, which were also the most fecund (Zimmerman et al. 1994).

In contrast to susceptibility, recovery from hurricane damage exhibited a clear, albeit variable pattern. At the individual repair level (i.e., growth and fecundity), adults from some species suffered relatively little damage and recovered rapidly (e.g., the late-successional $D$. excelsa and $L$. procera), others recovered rapidly from heavy damage (e.g., the pioneers A. floribunda and S. morotoni), whereas others exhibited weak recovery despite suffering heavy damage (e.g., C. arborea). Species recovery was also assessed as the response to enhanced resource availability after hurricanes. Favorable resource conditions (i.e., low crowding) increased the rate of diameter growth for all species. In general, the magnitude of this increase was greater for pioneer and late-successional species relative to secondary-forest species. Secondary-forest species such as C. arborea and $T$. heterophylla also exhibited weak positive fecundity responses to increased resources relative to other functional groups, although these patterns were highly variable.

Recovery of diameter growth and fecundity after storms may hinge on the degree to which individual species rely on sprouting as a means to regain lost biomass after disturbance. In a study of vegetative reproduction of trees in Barro Colorado Island, Paciorek et al. (2000) suggested that sprouting ability is poor for both the most shade-intolerant and most shade-tolerant species but variable for intermediate shade-tolerant species. Our results are consistent with this suggestion. Secondary-forest species such as $C$. arborea and C. sylvestris have high sprouting ability and appear to rely on sprouting to recover biomass rather than increasing diameter growth as a strategy to recover from disturbance. Pioneer species seem to have low sprouting ability in this analysis, possibly accruing a greater benefit from broad dissemination of seeds to new sites after damage, given the high likelihood that they will suffer severe damage during the storms (Zimmerman et al. 1994). In contrast to secondary-forest species, shade-tolerant species are unlikely to suffer complete canopy loss or tip-ups during storms (Zimmerman et al. 1994). As a result, they may be primed to take advantage of greater light levels within the canopy after storms by increasing both diameter growth rate and fecundity. This may be due to these tree species having better structural support (generally higher wood density) than secondary species and large labile carbon reserves that can be used for recovery after damage (Clark and Clark 1992). Given the important effects of hurricane regimes on forest structure and composition (Uriarte et al. 2009), these results suggest that differences in recovery rates among species may have a greater influence on forest dynamics than differences in the probability or effect of damage on trees (Putz and Brokaw 1989). Sprouting, in particular, can be the dominant pathway of regeneration for some species, representing an important axis of life history differentiation (Dietze and Clark 2008).

The range of responses to disturbance observed in this study do not entirely align with the life history characteristics associated with traditional ecological groupings of tropical tree species (Swaine and Whitmore 1988). Parallel research in grassland ecosystems during the past decade has also found that traditional classification schemes based on growth form or functional groups are not useful in predicting ecosystem function (Wright et al. 2006) or the response of species to environmental change (Reich et al. 2001, C. M. Clark et al. 2007). Recent research suggests that multiple traits, rather than membership in functional groups, may control the response of species to changing environmental conditions (Reich et al. 2001). Although functional groupings assume that these traits can be used to classify species, a large body of research calls the broad validity and consistency of this assumption into question (e.g., Craine et al. 2001, Wright et al. 2007). Understanding species responses to the changing resource environments that accompany disturbance will benefit from a traitbased approach that examines how species-specific variation in traits across environmental gradients modulates demographic rates (Webb et al. 2010).

\section{Life history trade-offs in response to disturbance}

Life history trade-offs in colonization and competitive ability, or in the ability of species to survive under low resource conditions and exploit the temporary resourcerich conditions that may be generated after a disturbance could contribute to the maintenance of tropical forest tree species diversity. As in previous studies (e.g., Hubbell and Foster 1986, Pacala et al. 1996), we found life history trade-offs between the ability of species to survive at low resource conditions (high crowding survival) and to exploit the temporary resource-rich conditions often generated in the wake of disturbance (low crowding) in accordance with the expectation of the successional niche hypothesis. Trade-offs between colonization ability and high crowding (low light) survival are also apparent in our data, as predicted by competition-colonization theory.

In a similar analysis of tree growth and mortality data across 11 plots in North Carolina, Clark et al. (2010) found weaker trade-offs in demographic responses to changes in resource availability than those found in this study. Several mechanisms may explain this difference. The inclusion of data from 11 plots in the Clark et al. 
(2010) study may have increased the range of environmental conditions experienced by species and, as a result, may have decreased the likelihood of finding trade-offs in species' aggregated responses. Examining life history trade-offs in individuals distributed across a natural environmental gradient may hinder the detection of trade-offs. Although plausible, this mechanism is unlikely to be the cause of the weaker trade-offs observed at the North Carolina sites because recent analyses of paired individuals, one in the experimental gap matched with one in the understory (J. S. Clark, unpublished data), both with pre- and posttreatment response, show similar patterns (i.e., weak trade-offs). A second, and more likely possibility, is that there are true differences in the expression of trade-offs that are driven by variation in environmental conditions among the two sites. One important difference between the two sites is seasonality, which may impose very different dynamics among tree species. The forest in Puerto Rico is largely aseasonal in temperature and precipitation (Zimmerman et al. 2007), potentially leading to more intense competition for resources and less room for temporal partitioning of resources. At higher latitudes, lower sun angles also reduce the contrast between light gaps and shaded understory (Ricklefs 1977). A second difference is the relationship between sprouting and other life history characteristics. Dietze and Clark (2008) found that the resprouting of North American tree species was only weakly correlated with other life history trade-offs, in contrast to the strong trade-offs encountered in this study. The generality of our conclusions may hinge on the frequency and extent of canopy disturbance, which is far greater at the Puerto Rico site.

Trade-offs, such as those demonstrated here, can help to promote diversity in the context of environmental variation, including disturbance (Loehle 2000). The detail of inference available here shows that species employ diverse strategies to withstand and recover from hurricane damage. These strategies depend on the degree to which individual species rely on sprouting, repair of adult damage, demographic responses (e.g., greater sapling growth) to enhanced resource availability after storms, or long-distance dispersal as recovery mechanisms (Bellingham 2000). It is possible that species exhibiting enhanced juvenile growth after storms do so as an alternative to repairing damaged adults, a strategy that may allow them to increase potential seed production from established reproductive adults. Similarly, allocation of resources to developing sprouts under the favorable resource conditions that typically follow storms may come at the cost of compensatory responses in adult fecundity or surviving stem diameter growth. Finally, some species rely on colonization of new sites as the primary recovery mechanism (e.g., T. heterophylla, the only wind-dispersed species in the set), whereas others employ mixed strategies (e.g., A. floribunda).

As with other studies before, our results lend support to the notion that tree life histories may obey multidimensional trade-offs (e.g., shade tolerance vs. ability to grow quickly in full sun or to disperse) that can mediate the maintenance of species diversity in forest ecosystems (Clark and Clark 1992). Unlike previous research, our study highlights the rich array of species responses to disturbance. As such, this study provides insights into coexistence mechanisms in forests subject to repeated disturbance. This is a critical need in a world in which the frequency and intensity of many types of global and human-driven disturbance are expected to increase (Clark et al. 2001).

\section{ACKNOWLEDGMENTS}

We thank M. Aponte Pagán and the Luquillo field crews who inventoried the plot. Three anonymous reviewers offered helpful suggestions for improving the manuscript. This work was supported by the Mellon Foundation, NSF grants DEB0087214 and DEB-0516066 to J. Thompson, J. K. Zimmerman, and M. Uriarte, DEB 0425465 to J. S. Clark, DEB-LTREB 0639393 to J. K. Zimmerman and J. Thompson, and grants from NSF to the Luquillo Long-Term Ecological Research Program. The U.S. Forest Service and the University of Puerto Rico provided additional support. L. S. Comita received partial support from a fellowship from the Earth Institute, Columbia University.

\section{Literature Cited}

Bellingham, P. J. 2000. Re-sprouting as a life history strategy in woody plant communities. Oikos 89:409-416.

Canham, C. D., J. Thompson, J. K. Zimmerman, and M. Uriarte. 2010. Variation in susceptibility to hurricane damage as a function of storm severity in Puerto Rican tree species. Biotropica 42:87-94.

Clark, C. M., E. E. Cleland, S. L. Collins, J. E. Fargione, L. Gough, K. L. Gross, S. C. Pennings, K. N. Suding, and J. B. Grace, 2007. Environmental and plant community determinants of species loss following nitrogen enrichment. Ecology Letters 10:596-607.

Clark, D. A., and D. B. Clark, 1992. Life-history diversity of canopy and emergent trees in a Neotropical rain forest. Ecological Monographs 62:315-344.

Clark, J. S. 1996. Testing disturbance theory with long-term data: Alternative life-history solutions to the distribution of events. American Naturalist 148:976-996.

Clark, J. S., et al. 2010. High-dimensional coexistence based on individual variation: a synthesis of evidence. Ecological Monographs 80:569-608.

Clark, J. S., S. R. Carpenter, M. Barber, S. Collins, A. Dobson, and J. A. Foley. 2001. Ecological forecasts: an emerging imperative. Science 293:657-660.

Clark, J. S., S. LaDeau, and I. Ibañez. 2004. Fecundity of trees and the colonization-competition hypothesis. Ecological Monographs 74:415-442.

Clark, J. S., M. Wolosin, M. Dietze, I. Ibanez, S. Ladeau, M. Welsh, and B. Kloeppel, 2007. Tree growth inference and prediction from diameter censuses and ring widths. Ecological Applications 17:1942-1953.

Comita, L. S., M. Uriarte, J. Thompson, I. Jonckheere, C. D. Canham, and J. K. Zimmerman. 2009. Abiotic and biotic drivers of seedling survival in a hurricane-impacted tropical forest. Journal of Ecology 97:1346-1359.

Craine, J. M., J. Froehle, G. D. Tilman, D. A. Wedin, and F. S. Chapin. 2001. The relationships among root and leaf traits of 76 grassland species and relative abundance along fertility and disturbance gradients. Oikos 93:274-285.

Devoe, N. N. 1989. Differential seeding and regeneration in openings and beneath closed canopy in sub-tropical wet forest. Dissertation. Yale University, New Haven, Connecticut, USA. 
Dietze, M. C., and J. S. Clark. 2008. Changing the gap dynamics paradigm: vegetative regeneration control on forest response to disturbance. Ecological Monographs 78:331-347.

Elerian, O., S. Chib, and N. Shephard, 2001. Likelihood inference for discretely observed non-linear diffusions. Econometrica 69:959-993.

Forero-Montaña, J., J. Thompson, and J. K. Zimmerman. 2010. Population structure, growth rates and spatial distribution of two dioecious tree species in a wet forest in Puerto Rico. Journal of Tropical Ecology 26:433-443.

Hubbell, S. P., and R. B. Foster, 1986. Biology, chance and history and the structure of tropical rain forest tree communities. Pages 314-329 in J. M. Diamond and T. J. Case, editors. Community ecology. Harper and Row, New York, New York, USA.

Loehle, C. 2000. Strategy space and the disturbance spectrum: A life-history model for tree species coexistence. American Naturalist 156:14-33.

Lugo, A. E., and J. K. Zimmerman. 2002. Ecological life histories. Pages 191-213 in J. A. Vozzo, editor. Tropical tree seed manual. Agricultural Handbook 721. USDA Forest Service, Washington, D.C., USA.

Metcalf, C. J. E., J. S. Clark, and S. M. McMahon. 2009. Overcoming data sparseness and parametric constraints in modeling of tree mortality: a new non-parametric Bayesian model. Canadian Journal of Forest Research 39:1677-1687.

Pacala, S. W., C. D. Canham, J. Saponara, J. A. Silander, Jr., R. K. Kobe, and E. Ribbens. 1996. Forest models defined by field measurements: estimation, error analysis and dynamics. Ecological Monographs 66:1-43.

Pacala, S. W., and M. Rees. 1998. Models suggesting field experiments to test two hypotheses explaining successional diversity. American Naturalist 152:729-737.

Paciorek, C. J., R. Condit, S. P. Hubbell, and R. B. Foster. 2000. The demographics of resprouting in tree and shrub species of a moist tropical forest. Journal of Ecology 88:765777.

Putz, F. E., and N. V. L. Brokaw. 1989. Sprouting of broken trees on Barro Colorado Island, Panama. Ecology 70:508512.

Reich, P. B., D. Tilman, J. Craine, D. Ellsworth, M. G. Tjoelker, J. Knops, D. Wedin, S. Naeem, D. Bahauddin, J. Goth, W. Bengtson, and T. D. Lee, 2001. Do species and functional groups differ in acquisition and use of $\mathrm{C}, \mathrm{N}$ and water under varying atmospheric $\mathrm{CO}_{2}$ and $\mathrm{N}$ availability regimes? A field test with 16 grassland species. New Phytologist 150: 435-448.

Ricklefs, R. E. 1977. Environmental heterogeneity and plant species diversity: A hypothesis. American Naturalist 111:376-381.

Smith, R. F. 1970. The vegetation structure of a Puerto Rican rain forest before and after short-term gamma irradiation.
Pages 103-140 in H. T. Odum and R. F. Pigeon, editors. A tropical rainforest: a study of irradiation and ecology at El Verde, Puerto Rico. Division of Technical Information, U.S. Atomic Energy Commission, Springfield, Virginia, USA.

Swaine, M. D., and T. C. Whitmore. 1988. On the definition of ecological species groups in tropical rain forests. Vegetatio 75:81-86.

Thompson, J., N. Brokaw, J. K. Zimmerman, R. B. Waide, E. M. Everham III, D. J. Lodge, C. M. Taylor, D. GarciaMontiel, and M. Fluet, 2002. Land use history, environment, and tree composition in a tropical forest. Ecological Applications 12:1344-1363.

Tilman, D. 1994. Competition and biodiversity in spatially structured habitats. Ecology 75:2-16.

Uriarte, M., C. D. Canham, J. Thompson, and J. K. Zimmerman. 2004. A neighborhood analysis of tree growth and survival in a hurricane-driven tropical forest. Ecological Monographs 74:591-614.

Uriarte, M., C. D. Canham, J. Thompson, and J. K. Zimmerman. 2009. Natural disturbance and human land use as determinants of tropical forest dynamics: results from a forest simulator. Ecological Monographs 79:423-443.

Vandermeer, J., D. Boucher, I. Perfecto, and I. Granzow de la Cerda. 1996. A theory of disturbance and species diversity: evidence from Nicaragua after Hurricane Joan. Biotropica 28:600-613.

Walker, L. R. 1991. Tree damage and recovery from Hurricane Hugo in Luquillo Experimental Forest, Puerto Rico. Biotropica 23:379-385.

Webb, T. C., J. A. Hoeting, G. M. Ames, M. I. Pyne, and N. L. Poff. 2010. A structured and dynamic framework to advance traits-based theory and prediction in ecology. Ecology Letters 13:267-283.

Wright, I. J., et al. 2007. Relationships among ecologically important dimensions of plant trait variation in seven Neotropical forests. Annals of Botany 99:1003-1015.

Wright, J. P., S. Naeem, A. Hector, C. Lehman, P. B. Reich, B. Schmid, and D. Tilman, 2006. Conventional functional classification schemes underestimate the relationship with ecosystem functioning. Ecology Letters 9:111-120.

Wright, S. J. 2002. Plant diversity in tropical forests: a review of mechanisms of species coexistence. Oecologia 130:1-14.

Zimmerman, J. K., E. M. Everham, R. B. Waide, D. J. Lodge, C. M. Taylor, and N. V. L. Brokaw. 1994. Responses of tree species to hurricane winds in subtropical wet forest in Puerto Rico: implications for tropical tree life histories. Journal of Ecology 82:911-922.

Zimmerman, J. K., S. J. Wright, O. Calderón, M. A. Pagan, and S. Paton. 2007. Flowering and fruiting phenologies of seasonal and aseasonal Neotropical forests: the role of annual changes in irradiance. Journal of Tropical Ecology $23: 231-251$.

\section{Supplemental Material}

Appendix A

Mapped stand used for demographic inference (Ecological Archives E093-018-A1).

Appendix B

Parameters and state variables included in the models (Ecological Archives E093-018-A2).

Appendix C

Selection of priors (Ecological Archives E093-018-A3).

Appendix D

Model evaluation (Ecological Archives E093-018-A4). 